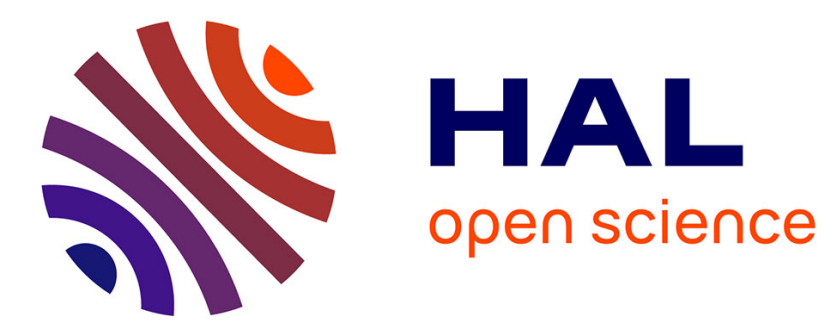

\title{
De la place des médias dans l'analyse des affaires sportives
}

Henrique Rodas, Patrick Trabal

\section{To cite this version:}

Henrique Rodas, Patrick Trabal. De la place des médias dans l'analyse des affaires sportives. Le Temps des médias. Revue d'histoire, 2008, 9 (9), pp.91-106. 10.3917/tdm.009.0091 . hal-01471537

\section{HAL Id: hal-01471537 \\ https://hal.parisnanterre.fr/hal-01471537}

Submitted on 2 Dec 2020

HAL is a multi-disciplinary open access archive for the deposit and dissemination of scientific research documents, whether they are published or not. The documents may come from teaching and research institutions in France or abroad, or from public or private research centers.
L'archive ouverte pluridisciplinaire HAL, est destinée au dépôt et à la diffusion de documents scientifiques de niveau recherche, publiés ou non, émanant des établissements d'enseignement et de recherche français ou étrangers, des laboratoires publics ou privés. 


\section{De la place des médias dans l'analyse des affaires sportives}

\section{Henrique Rodas ${ }^{\star}$, Patrick Trabal $^{\star \star}$}

Depuis plusieurs années, des chercheurs ont mobilisé les apports de la sociologie morale et politique (Boltanski et al.,1984,Boltanski,1990,Boltanski et Thévenot,1991) pour étudier les affaires judiciaires qui concernent le sport (Duret et Trabal, 2001, Trabal, 2006). Il s'agit d'analyser les témoignages et les accusations, les jeux d'expertises et les processus d'authentification, les décisions et leurs critiques, pour saisir les façons dont les acteurs s'inquiètent des injustices dans le sport, dénoncent certaines pratiques, se défendent et retournent les accusations, légiferent et s'accordent (ou non) sur des principes d'action. La presse joue un rôle souvent central dans ces disputes, et ce d'autant plus que la discipline est médiatisée.

D'une part, on s'interrogera sur les modalités de traitement des affaires sportives. On s'efforcera de préciser comment la presse entre dans l'arène et comment elle se détache des enjeux de l'épreuve et des résultats sportifs pour s'attacher à suivre les disputes, les différends et autres litiges extra-sportifs.
D'autre part on s'attachera à préciser les liens que les journalistes entretiennent avec les autres protagonistes et comment leurs relations entre connivence et tension participent à l'évolution de l'affaire. Quelle est la nature du travail journalistique et en quoi les types d'enquêtes et d'investigations menées modifient-ils le déroulement des polémiques?

Cet article se propose de contribuer à une analyse des médias qui cherchera non pas à s'intéresser aux contraintes du journaliste (Lemieux, 2000) dans son activité ordinaire, mais à qualifier les effets du travail de la presse dans la dynamique des affaires dites sportives.

\section{Le logiciel PROSPERO ${ }^{\circledR}$ pour l'analyse de deux affaires sportives}

Pour clarifier ces questionnements, nous porterons l'analyse sur deux affaires dont les degrés de médiatisations different. Le Tour de France 1998, qui se confond avec «l'affaire Festina ", a été marqué par la mise en

${ }^{\star}$ Laboratoire Sport \& Culture (Université Paris X).

${ }^{* *}$ GSPR (EHESS). 
évidence du dopage comme pratique non seulement courante dans le peloton mais aussi largement organisée par les équipes cyclistes. Quelques jours avant le départ de l'épreuve, l'arrestation d'un soigneur de l'équipe Festina en possession de 400 produits dopants, débouche sur une série d'interpellations et des coureurs sont empêchés de poursuivre la course. D'autres équipes sont mises en cause et, à la suite d'arrestations et d'abandons lors d'une étape en Suisse pour échapper à la police française, c'est un peloton très réduit qui franchit la ligne d'arrivée à Paris. "Il faut arrêter ce Tour " demande alors Jean-Marie Colombani à la une du Monde. Son éditorial comme le travail d'enquête de journalistes dans les poubelles d'un hôtel où résident les cyclistes - reportage à l'origine d'une grève de l'ensemble des coureurs - rappellent que le déroulement de cette affaire n'est pas détachable de sa médiatisation. Très vite, le récit gagne à se nourrir d'autres points de vue avec plusieurs ouvrages publiés par des journalistes refaisant l'enquête et les témoignages publiés par les protagonistes eux-mêmes dans des autobiographies.

Le deuxième cas étudié concerne une affaire moins connue. Depuis près de trois décennies, les flux migratoires de footballeurs étrangers, africains pour la plupart, vers l'Union européenne se sont intensifiés et, régulièrement, sont dénoncées les pratiques "négrières " de dirigeants de clubs, d'agents de spor-tifs ou de personnes se déclarant comme tels. Le rêve d'un jeune Africain, s'imaginant en star mondiale du football se transforme souvent en une dure réalité: la vie d'un "sans papier ». Alertée par le cas d'un jeune reconduit à la frontière, la ministre de la jeunesse et des sports, Marie-George Buffet, lance en septembre 1999 une enquête au niveau national qui donne lieu à un rapport sur les conditions de recrutement, d'encadrement et de suivi des jeunes dans les centres de formation. Dans un contexte difficile marqué par des affrontements politiques sur la question de l'immigration, le « rapport Donzel ", du nom de l'Inspecteur Général en charge de l'enquête, publié trois mois plus tard, souligne plusieurs défaillances et pointe des pratiques qui ne respectent ni la réglementation sportive (du football en particulier), ni les textes officiels de l'Éducation nationale, ni les lois sur l'immigration de l'État français. Ce texte explicite les responsabilités des uns et des autres, et malgré son classement " confidentiel ", il est l'objet d'une fuite dans la presse. À la suite d'un premier papier dans $L e$ Parisien du 25 janvier 2000 dénonçant les sureffectifs dans les centres de formation et soulignant en particulier la présence clandestine de jeunes Africains, une série d'articles lance un débat qui, dans ses plus simples contours, oppose le monde du football aux arguments développés dans l'étude ministérielle. De façon schématique, dans leurs articles, les journalistes reproduisent d'abord les formules chocs ${ }^{1}$ et les orientations du rapport, pour ensuite 
donner place aux réponses véhémentes des acteurs du football.Jusqu'à la conférence de presse du 14 février 2000 réunissant la ministre et les dirigeants des instances du football français (la Fédération Française de Football et la Ligue Nationale du Football), conférence qui a pour but d'afficher publiquement une cohésion entre les parties et de communiquer les dispositions amorcées par la loi dite Buffet du 28 décembre 1999, ce sujet entraîne une floraison d'articles (environ 70 répertoriés) avant d'être peu à peu délaissé et oublié par les médias.

Ces deux dossiers ont été choisis pour des raisons de proximité et de distance, car s'ils sont de nature différente, ils se déploient néanmoins à peu près au même moment, sous le même gouvernement en engageant des acteurs semblables: athlètes de haut niveau et responsables sportifs évoluant dans des disciplines populaires et professionnelles, autorités publiques mais aussi d'autres États, institutions internationales (fédérations sportives, Union européenne...). Pour répondre à nos questions sur le rôle des médias, nous avons constitué pour chacune de ces affaires, deux corpus :l'un est composé des productions des journalistes, l'autre des contributions d'autres acteurs.

Sur l'affaire Festina, nous avons donc rassemblé les articles de presse publiés entre 1998 et 2001 dans trois quotidiens nationaux (Libération, Le Monde, Le Figaro), un quotidien régional (SudOuest), et le quotidien sportif L'Equipe, ainsi que les résultats de leurs investi- gations publiés sous la forme de quatre ouvrages. Symétriquement, nous avons regroupé les témoignages des différents protagonistes. Cette opération a été facilitée par le fait que les récits de plusieurs cyclistes, du soigneur, du directeur sportif, de l'organisateur de l'épreuve et du responsable de la Fédération ont été édités dans onze livres (nous avons retenu les chapitres concernant cette affaire).

De la même façon, le dossier des migrations de footballeurs étrangers a donné lieu à la construction de deux sous-corpus. L'un composé de 119 articles de presse parus entre février 1996 et décembre 2006 et émanant de 23 supports différents ${ }^{2}$. L'autre souscorpus rassemble les productions hétérogènes de différents acteurs, à savoir des textes législatifs, des documents parlementaires, des rapports des ouvrages bibliographiques, et des entretiens.

Pour faire face au volume de données, nous avons eu recours au programme Prospéro. Conçu et réalisé par Francis Chateauraynaud et Jean-Pierre Charriau, le logiciel PROSPERO ${ }^{\circledR}$ (PROgramme de Sociologie Pragmatique Expérimentale et Réflexive sur Ordinateur $^{3}$ fut construit en tirant partie d'une confrontation des apports de la statistique textuelle (calculs des fréquences et des co-occurrences multiples), de la linguistique (analyse des énoncés et des enchaînements discursifs), de l'intelligence artificielle (système de raisonnements et d'inférences), des analyses de réseaux (calculs 
d'associations et de graphes) et de la sociologie cognitive (étude des procédés de codage et d'interprétation). Il permet la description et l'analyse de dossiers complexes, composés de discours hétérogènes et provenant d'un grand nombre d'acteurs.

\section{Festina : une belle affaire pour les médias}

En séparant les productions des journalistes de celles émanant des autres protagonistes (coureurs, soigneurs, directeur sportif...), nous avons cherché à recenser les variations significatives du lexique ${ }^{4}$ afin de repérer si l'univers déployé par la presse différait ou non de celui des autres acteurs. Le résultat de cette opération de partitionnement fait apparaitre que le souscorpus journalistique est particulièrement marqué par la judiciarisation de l'affaire.La forte présence des noms des personnages centraux (Voet, Roussel, Keil...), de leurs fonctions (soigneur, directeur sportif, juge...), la multiplicité des institutions (FFC, UCI, ASO ... $)^{5}$ et surtout la sur-représentation du vocabulaire judiciaire (mise en examen, garde à vue...) et des qualifications juridiques (" incitation et facilitation à l'usage et à l'emploi de substances dopantes ", « importation, offre et cession de substances vénéneuses "...) conduisent à penser que les médias traitent ces faits comme une affaire et non comme une simple dispute sur un événement sportif (Duret et Trabal, 2001). Symétriquement, l'analyse du répertoire des autres protagonistes rend visible les marques classiques du témoignage: les catégories "vie quotidienne " et "parler populaire " jouxtent celles renvoyant à l'expérience cycliste, avec ses luttes (on parle de bagarres, de "bastons ") et ses grandeurs ainsi qu'aux valeurs morales que cette affaire met à l'épreuve. Le propos s'articule le plus souvent autour de récits décrivant l'univers de vie des protagonistes - à la fois banal et extraordinaire - dans lequel se déroule une intrigue. À un premier niveau, on peut donc dire que ce qui distingue les productions des journalistes de celles des autres protagonistes tient aux types de description des événements. Ceux-ci surgissent dans le quotidien des personnes impliquées et l'effet de surprise ainsi suscité est d'autant plus fort que le récit fait place à une dramatisation inattendue ${ }^{6}$. Ces mêmes événements sont relatés avec une certaine distance par les journalistes qui n'hésitent pas à mobiliser massivement le vocabulaire juridique pour qualifier les faits et le déploiement des positions institutionnelles pour raconter les rebondissements de l'affaire.

Ces constats méritent toutefois trois nuances.D'abord,le répertoire d'un des protagonistes, Daniel Baal - président de la Fédération Française de Cyclisme mis en examen par le juge ${ }^{7}$ - est plus marqué par des logiques médiatiques et gestionnaires, d'une part, et par des figures dénonciatrices, d'autre part. Cela peut s'expliquer par le statut institutionnel de l'intéressé souvent décrit 
comme un " communicant ". On constate ensuite une différence entre les ouvrages et les articles: les rebondissements judiciaires y cèdent la place à des descriptions des techniques de dopage et d'usage de produits dopants. Cette propriété tient sans doute plus au format livresque qui laisse plus d'espace pour des précisions sur les pratiques prohibées. Enfin, le cas de L'Equipe, mérite d'être distingué :les événements du Tour 1998 et les descriptions des performances cyclistes sont traités séparément, alors que les titres des articles jouent sur la proximité entre l'épreuve sportive et les épreuves que l'affaire impose aux coureurs: « le moment de vérité » (27 juillet 1998), "l'orgueil d'un grand " ou "l'honneur retrouvé " (29 juillet 1998) renvoient en fait aux rebondissements de la course, dans le haut du classement.

Pour saisir les rapports entre les journalistes et les autres protagonistesauteurs, on peut recenser les façons dont ces derniers qualifient le travail des médias. On s'est intéressé aux différentes formes pour désigner les journalistes (journaliste, reporter, chroniqueur...) et leurs productions (émission, article, édito...). Surgissent alors deux registres:l'affaire et ses rebondissements judiciaires d'une part, les modes de dénonciation d'autre part. Ce dernier point mérite d'être examiné plus finement. Comme nous l'avons déjà souligné (Duret et Trabal, 2001), la plainte envers les médias a jalonné l'histoire récente du dopage et s'est souvent réglée devant des tribu- naux saisis pour diffamation. Rien de très nouveau donc dans ces dénonciations des pratiques journalistiques, si ce n'est qu'elles se déroulent dans un contexte où ce sont les sportifs et leurs encadrements qui se trouvent poursuivis par la Justice. On ne trouve aucune trace dans le corpus d'une accusation selon laquelle les journalistes seraient animés par un esprit de revanche, mais on repère plusieurs marques de rupture sur les rapports journaliste/sportif que cette affaire a généré. Ces changements sont de plusieurs natures.

À un premier niveau, il convient de souligner que des journalistes non spécialistes du sport entrent dans l'arène. Non soumis à cette habitude de célébration des exploits sportifs à laquelle leurs confrères s'adonnent souvent, ils mobilisent des méthodes d'investigation déstabilisantes pour le monde du cyclisme. Du coup, les journalistes sportifs changent aussi le ton ce qui conduit des sportifs à critiquer amèrement ce basculement, à l'instar de Richard Virenque s'en prenant, dans son ouvrage, au rédacteur en chef de L'Equipe Magazine:

J'avais toujours eu avec lui des rapports nets et sans ombre. Jamais, je ne lui avais refusé une interview. Et jamais, je n'avais refusé la moindre de ses invitations lorsque le calendrier me le permettait. Il semblait avoir pour moi, à l'époque, une certaine sympathie. Il se disait amateur de cyclisme. Je n'en doutais pas. Je ne le savais pas, en revanche, si peu doué de courage, et si apte à enterrer les amis d'hier. (Virenque, 1999) 
De même, à la suite de plusieurs "reportages-poubelles » (sic) dans lesquels des équipes de télévision s'emploient à filmer les déchets laissés par les équipes cyclistes dans les hôtels, les coureurs menacent de se mettre en grève. C'est Jean-Marie Leblanc, directeur du Tour de France au moment des faits qui utilisera son double statut d'ancien cycliste et d'ancien journaliste pour trouver un compromis et sauver cette édition de la Grande Boucle en reprenant à son compte, in fine, les vindictes des coureurs. Ce sont aussi des équipes sportives, dont l'emblématique Festina qui s'en prennent aux journalistes: le propriétaire de cette marque de montres obtient un droit de réponse à la suite d'un papier peu flatteur. Mais les tensions entre le monde cycliste et la presse dépassent le traitement de l'affaire. Ainsi, on apprend que deux journalistes de la télévision publique danoise «sont privés de Tour de France » en raison d'une enquête mettant en cause l'icône nationale, Bjarne Riis, ex-vainqueur du Tour.

Les images étaient éloquentes. Le Prix du silence démarrait par une séquence filmée depuis une voiture stationnée devant un hôtel de Biseglia, en Italie, en plein Tour des Pouilles en septembre 1998. Après le départ des coureurs de l'équipe Ros Mary, on aperçoit le masseur, un sac plastique vert à la main, se diriger vers des conteneurs placés dans la rue. Quelques instants plus tard,il repart, les mains vides. Après avoir attendu dix minutes, les deux journalistes dirigent leur caméra vers les conteneurs. Le sac plastique est enfourné dans un carton. Les journalistes s'en emparent et en examinent le contenu à l'écart: 17 seringues sanguinolentes, 13 médicaments différents et une ampoule dont l'étiquette a été soigneusement arrachée. L'analyse d'un laboratoire de Copenhague révélera des traces d'EPO, ce produit qui augmente artificiellement le nombre de globules rouges dans le sang. (Libération, 3 juillet 2000).

La construction de catégories a permis de repérer les jeux de dénonciations et d'accusation, et de recenser les fondements de ces critiques croisées entre la presse et le monde cycliste. Ce dernier s'efforce de défendre le sport et dénonce le fait que l'on s'intéresse plus au dopage qu'au vélo ; les journalistes, selon les coureurs, préfereraient assurer leurs carrières professionnelles et fouiner pour révéler quelques scandales plutôt que de relayer la passion du cyclisme. Symétriquement, la presse défend la nécessité de livrer une information complète et s'offusque des pressions du monde cycliste qui «ne constituent pas un traitement équitable auquel les coureurs ont droit et auquel l'ensemble du public est attaché ". (Le Monde, 25 septembre 1998).

Ces jeux de critiques croisées se donnent à lire sur des supports différents. C'est dans les ouvrages que les protagonistes s'indignent des pratiques des médias. Parce qu'ils disposent d'un espace pour déployer les contraintes de leurs métiers (Buisine, 2007), les sportifs et leur encadrement peuvent à la fois préciser les relations "normales " 
qu'ils entretiennent avec la presse (par exemple, le jeu des interviews à la fin d'une compétition) et mettre en exergue les changements provoqués par cette affaire. Les journalistes, quant à eux, s'expriment à la fois dans leurs médias et dans des ouvrages où ils dévoilent la face jusqu'alors "cachée " des interactions avec les acteurs du monde cycliste. Ces dernières informations recoupent partiellement celles que l'on trouve dans les autres récits. Il n'y a donc, dans les livres, pas de différences notables entre les journalistes et les autres protagonistes: les argumentations et les jugements sont intrinsèquement liés aux descriptions des différentes contraintes des «métiers » respectifs (journaliste, coureur, soigneur...), et les pressions auxquelles sont soumises les cyclistes peuvent être mises en équivalence avec celles s'exerçant sur les journalistes. Il reste que ces derniers bénéficient d'un accès permanent et donc privilégié à l'espace public, ce qui leur permet de dénoncer, quand ils le souhaitent, les opérations d'intimidation dont ils sont l'objet.

\section{L'épreuve de l'interview}

Mais c'est sur un moment particulier que nous souhaitons porter l'attention. L'interview, qui en dehors des moments de crise, fait partie de la routine du travail journalistique et de l'exercice du métier sportif de haut niveau, apparait comme un moment d'épreuve, c'est-à-dire qui entraîne potentiellement un changement d'état (Chateauraynaud, 1991). Il s'agit en effet d'une confrontation directe entre les acteurs de l'affaire et le journaliste. C'est un moment redouté où l'on peut perdre la face et, tout comme dans une rencontre sportive, il convient de s'entendre sur les règles de l'épreuve. Mais le match est intrinsèquement inégal car, si aucun accord n'est trouvé, les journalistes gardent la possibilité d'informer le public des raisons du désaccord $^{8}$.

Les interviews qui laissent apparaître des critiques croisées sont donc très rares. Les «questions qui fầchent » sont généralement posées en reprenant les accusations d'autres protagonistes et les indignations contre les journalistes restent peu nombreuses ${ }^{9}$. Dans la majorité des cas, l'interview permet à un protagoniste de régler ses comptes avec un autre, ce qui contribue à la dynamique de l'affaire. Du coup, des parallèles et des rapprochements entre l'interview d'un journaliste et l'interrogatoire des policiers sont possibles. Pour les enquêteurs comme pour les journalistes, il s'agit de faire parler. Et pour un collectionneur de témoignages visant à établir la vérité des pratiques, il existe de "bons clients». Ceux-ci ont une double qualité.D'une part, en parlant, ils accusent, ce qui permet de relancer l'affaire et de nourrir la production journalistique. D'autre part, leur position - ce sont généralement le cas des repentis ou des cyclistes militants de l'anti-dopage qui ont une bonne connaissance des pratiques du peloton -, conduit à dévoiler des pra- 
tiques, à briser la loi du silence, à rendre publiques les choses que d'aucuns s'efforcent de masquer. Cela revient le plus souvent à affirmer la forte présence du dopage et à conforter la conviction longtemps partagée par les journalistes qui, faute de preuves étayées et de témoignages solides, se retrouvaient en difficulté.

C'est chose entendue depuis l'année dernière que la maladie est sur le troupeau. Ces dernières sont gardées par des chiens de berger (les directeurs sportifs). Mais il y a quand même quelques bêtes saines. Elles passent une tête dans l'enclos. On les reconnaît facilement, car la main du journaliste peut les caresser sans risque. C'est évidemment exagéré, mais il y a de ça dans ce manège quotidien qui tient de la foire aux bestiaux. Christophe Bassons (...) n'a jamais refusé l'aumône d'une interview.

Les rapports entre les journalistes et les acteurs de l'affaire peuvent ainsi se répartir grossièrement en deux types. Dans un cas, on est dans une logique de la révélation qui assure conjointement des succès de presse et la confirmation que le combat mené depuis des années était fondé. Dans l'autre, on bascule dans le registre de l'enquête, en recoupant les informations, en fouillant dans les valises des équipes sportives, en tentant d'obtenir des aveux. La question cruciale que posent ces types d'activité journalistique concerne la complexité du dopage qui ne peut pas se résumer à une association entre l'honnêteté des protagonistes et leur prolixité.

\section{Les médias au secours} des "sans-papiers du football "

Dans le cas de l'immigration des footballeurs africains, la distribution temporelle des deux sous-corpus fait apparaître une différence de chronologie et de régularité des publications: les textes des non-journalistes sont davantage étalés dans le temps et postérieurs à la majorité des articles de presse. Ces derniers font une large place au registre de l'alerte (Chateauraynaud et Torny, 1999) mais aussi aux questions de clandestinité, d'esclavagisme, de dénonciation des mouvements de migration de footballeurs et des centres de formation "parallèles ". L'autre sous-corpus est marqué par des descriptions liées aux univers de vie des acteurs (le championnat, les efforts physiques...) et aux réglementations organisant le monde du football.Ainsi, tout comme dans l'affaire Festina, la presse met moins l'accent sur le monde sensible des acteurs et, lorsque se déploie le registre de l'investigation, c'est essentiellement pour relater "l'enquête" ministérielle et sa critique.

$\mathrm{La}$ version publique du rapport Donzel ${ }^{10}$ joue un rôle central dans ce dossier.Le rapport se situe à la frontière de deux logiques: c'est à la fois un document officiel visant à orienter une politique et un recueil de données fondée sur une enquête de terrain sur lequel vont s'appuyer les journalistes. Contrairement à l'affaire du Tour 98 qui a conduit les journalistes à s'engouffrer dans la brèche ouverte par les 
policiers et le juge et mener leurs investigations, le travail de la presse est ici d'une autre nature. En effet, alors que le premier dossier est étoffé, épisode après épisode, d'aveux, de recoupements et de preuves directes de l'ampleur des pratiques dopantes, la polémique sur les mouvements migratoires des footballeurs ne se nourrit que de l'opposition entre la dénonciation publique et les réactions défensives du monde du football:

"Autant dire que cette «bombe médiatique " a aussitôt suscité une levée de boucliers, comme [le club de] Toulouse, directement mis en cause, dont le président, Jacques Rubio, s'est empressé de démentir les dites informations. " (Foot Magazine, $1^{\mathrm{er}}$ mars 2000).

Pour dénoncer l'injustice et rendre tangibles les faits, certains journalistes convoquent des victimes. Le cas Serge Lebri occupe ici une place décisive. La presse cite le rapport ministériel et développe souvent l'exemple de la situation emblématique de ce jeune footballeur ivoirien expulsé du territoire national.

Comment les journalistes mobilisent-ils le récit des victimes et/ou le propos des "persécuteurs " (Boltanski et al.,1984) pour évaluer les critiques? Le logiciel a permis de recenser l'ensemble des cas explicitement dénommés de jeunes étrangers victimes en France. Parmi les 119 articles composant notre corpus presse, seulement trente-quatre évoquent la vie de ces jeunes footballeurs au nombre de dix. Cinq d'entre eux étant sur le chemin de la réussite sportive puisqu'ils ont signé un contrat avec leur club, les médias se concentrent sur cinq victimes avérées, au premier rang desquelles Serge Lebri (ou Serge X) ${ }^{11}$. Les victimes sont donc peu présentes dans la presse, sans doute en raison des difficultés spécifiques à ce type d'investigation et des problèmes que pose l'anonymat.

Les descriptions des parcours de ces footballeurs répondent à un même format. Chaque sportif est tout d'abord convoqué pour son " histoire ». Le 19 octobre 1996, Libération titre par exemple: «L'histoire à rebonds de Lassissi. D'Abidjan à Rennes, itinéraire d'un footballeur doué mais pas toujours gâté ». Les cas sont alors contextualisés à partir d'un survol des étapes significatives de la carrière du jeune: l' " enrôlement » à l'adolescence par un «agent douteux » motivé par des intérêts financiers; 1 'abus des clubs professionnels et amateurs qui ne prennent pas en charge la régularisation administrative de l'enfant avec les risques d'une précarisation puis l'abandon et parfois une reconduite à la frontière à sa majorité. De ces articles sont absentes les alternatives possibles pour ces jeunes (choix d'autres agents, dirigeants, etc...), et surtout leurs dénonciations directes des contraintes qu'ils subissent. Néanmoins, l'exemplification de ces carrières typiques met à nu le dispositif organisé de la migration des joueurs africains. 
La question de l'ampleur du phénomène se pose. Plusieurs acteurs s'efforcent de dénombrer les "footballeurs sans papiers " mais l'entreprise s'avère difficile. Le rapport cite des chiffres partiels relatifs à quelques clubs. Le Parisien du 25 janvier 2000 parle d'une centaine de cas; le lendemain l'Humanité cite Le Parisien en faisant disparaître l'approximation. Lors de nos entretiens réalisés au ministère, aucun chiffre n'est clairement affirmé: "On pouvait remplir l'équivalent de trois avions charters ", "ça touchait environ 3000 personnes si on associe les proches »... Les approximations perdurent au-delà de cette période polémique. Lors d'une autre campagne d'entretiens que nous avons réalisée en 2007 dans le monde associatif, on évalue à "plusieurs centaines " le nombre de jeunes concernés sans que cette estimation puisse être plus précise ou même vérifiée.

Parallèlement, les journalistes se tournent vers les acteurs désignés dans le rapport comme les responsables. Le travail de la presse consiste alors à pousser les portes des centres de formation pour recueillir les réactions des dirigeants. Ces derniers contestent les résultats de l'enquête, soit en critiquant l'interprétation des chiffres qu'ils avaient confiés au Ministère, soit en relativisant l'ampleur du phénomène. À l'occasion d'un entretien, Jacques Donzel nous a exposé l'ensemble des moyens qu'il avait employés pour approcher au mieux le nombre de ces jeunes. Il a procédé par recoupements d'indices: traçage des cars employés pour le ramassage scolaire, croisement des listes des demandeurs d'une carte de séjour en préfecture et des licences déclarées à la Fédération Française de Football. Aucune de ces informations susceptibles de consolider les données du rapport n'apparaît dans la presse.

Le propos des dirigeants français sur la fiabilité de l'enquête a eu pour but de mettre un terme au débat plutôt que de le relancer. Cette absence de réactions permet aux "persécuteurs » de se présenter comme des « victimes»; elle renvoie aussi à la complexité des rapports que le monde du football entretient alors avec le ministère.

L'ampleur du phénomène et le dénombrement des footballeurs concernés étaient déjà au cœur des préoccupations de la Ministre. Une des principales mesures annoncées par Marie-George Buffet, le jour de la fuite dans la presse du rapport (25 janvier 2000), revenait à nommer dans chaque préfecture un service chargé de contrôler la situation des jeunes joueurs et des centres de formation des clubs et ainsi de créer un dispositif pour les recenser. Cette volonté de contrôler le football n'est pas toujours perçue de façon positive.Ainsi,l'édition du 15 février de L'Equipe est-elle particulièrement vindicative et critique:

Le Rapport ministériel sur la formation a fait beaucoup de bruit pour aboutir à des mesures dirigistes renforçant les contrôles sur les centres de formation, déjà très surveillés. (L'Equipe, 15 février 2000). 
Les missions de ces services préfectoraux ne sont pas sans poser problème. Un seul journaliste pousse l'enquête dans ces administrations et rapporte le témoignage d'un fonctionnaire en charge des questions liées à l'immigration (Sud Ouest, 27 janvier 2000) qui souligne la complexité du suivi des "sans-papiers". L'agent lui indique tout d'abord que «le monde du football ne bénéficie d'aucun passedroit ". Mais après avoir exposé les types de situations irrégulières que les services préfectoraux géraient, il décrit des situations plus difficiles à appréhender:

«S'ils sont entrés en situation irrégulière, après se pose un autre problème, dès lors qu'ils passent d'un club à l'autre. Ils doivent avoir une couverture sociale. Mais, perdus de vue de club en club, ils peuvent même tomber dans le droit commun en étant considérés comme mineurs en danger. "

Et le journaliste d'ajouter: «On le voit, les services préfectoraux se retrouvent devant un cas de figure qui les plonge dans une certaine perplexité.» Il montre ainsi que ces services n'ont pas la capacité de faire face aux mécanismes sous-jacents de l'immigration clandestine du football. En dehors de cette investigation, les médias ne sont pas allés au-delà de la polémique lancée par les dirigeants et n'ont pas poursuivi l'enquête sur la fiabilité des données du rapport.

\section{Un dossier traité à l'échelle européenne}

Les jugements sur le travail journalistique restent assez contrastés. L'universitaire Evariste Tshimanga Baka-

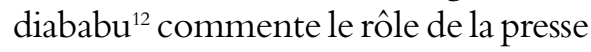
belge et française dans l'évolution de la prise de conscience du phénomène en Europe.

"Ces articles avaient pour but d'informer l'opinion tant nationale qu'internationale sur les pratiques abominables [...] en tendant à briser la loi du silence sur ces pratiques éhontées qui sont enregistrées dans des pays qui sont censés défendre avec virulence les droits de l'homme [...]. » Et d'ajouter: «Si à cette époque la France semblait loin de ces pratiques inadmissibles, aujourd'hui c'est chose faite [...]. »

L'auteur à travers sa critique souligne l'autonomie et les rôles informatif et décisif des médias dans cette affaire et leur concède un rôle d'acteurs à part entière. Les protagonistes du dossier partagent parfois ce point de vue sur l'indépendance des médias mais en regrettant certaines conséquences. C'est le cas du président de la Ligue Nationale de Football (LNF) lors de la conférence de presse organisée par les instances du football du 10 février 2000:

"J'ai eu en main le rapport de quarante pages du ministère de la Jeunesse et des Sports il y a trois semaines, et je ne l'ai pas trouvé si désobligeant que ça ", a déclaré Noël Le Graët qui a regretté l'exploitation négative qui en a été faite, selon lui, par la presse.» (Le Figaro, 12 février 2000). 
En défendant le rapport et son auteur, ce responsable du football français accuse les journalistes. Il rejoint, en cela, la position de la Ministre - ce qui revient à pacifier les rapports entre les instances du football et les pouvoirs publics. MarieGeorge Buffet emploie, elle aussi, ce procédé, à des fins identiques:

«J'ajoute que les réactions, parfois très vives, de certains clubs, ont été provoquées par des articles de presse avant la publication du rapport lui-même.Je n'avais donc pas à les commenter. " (extrait de la conférence de presse ${ }^{13}, 14$ février 2000).

Cette enquête ministérielle était annoncée par Marie-George Buffet dès le 15 septembre 1999 et les journalistes commencent dès novembre 1999 à être attentifs aux situations dans d'autres États européens. La presse française évoque au même moment la parution d'un rapport du Sénat italien faisant état de 5282 footballeurs extra-communautaires âgés de moins de 16 ans employés par des clubs amateurs. Comme en France, les pouvoirs publics ont mené l'enquête. Et c'est le récit de vie d'un jeune ivoirien de 14 ans qui a joué le rôle de déclencheur dans la presse.Toutefois, l'enquête ne rencontre pas un franc succès auprès des instances concernées. Les journaux français font également écho à deux autres scandales qui éclatent en Italie à la mi-janvier 2000 , soit quelques jours avant la fuite du rapport. En Belgique, le problème des "sans-papiers " du football est dénoncé depuis 1997 par le Centre pour l'égalité des chances et la lutte contre le racisme (dont les revendications avaient eu un écho auprès de dirigeants de l'Union royale belge de football à la suite de la projection du film de Manu Riche ${ }^{14}$ au festival de cinéma de Bruges en mars 1998), et par l'association belge "Sport et Liberté ». On rapporte aussi le cas des Pays-Bas où le Service d'immigration et de naturalisation (IND) a identifié dix-huit footballeurs étrangers en situation irrégulière dans des clubs professionnels.

Le synchronisme des événements met en avant la dimension européenne du problème.Dans la presse hexagonale, ces faits ne sont pas systématiquement mis en relation avec l'affaire en France.

Ainsi, le travail qui consiste à mettre en série, à relier et à comparer les situations dans les différents États, sert-il davantage à souligner la dimension internationale de la question qu'à relativiser le cas français. La succession des affaires génère l'idée de l'existence de filières clandestines de recrutement au sein des pays de l'Union européenne qui appelle à la mise en œuvre de politiques dépassant les cadres nationaux.

Quelques semaines plus tard, la présidence de la France à l'Union européenne en juillet 2000 donne l'occasion de travailler à l'élargissement de l'action de la Ministre des sports.

Les choses pourraient aller très vite: Marie-George Buffet ne cache pas son désir de parvenir à un résultat lors de la conférence intergouvernementale qui aura lieu à Paris en décembre, sous présidence française. (Le Monde, 17 mars 2000). 
Dans cette dynamique, en juillet 2001, à la suite de confrontations avec la Commission européenne, la Fédération Internationale de Football (FIFA) édicte un nouveau règlement sur les transferts qui distingue les footballeurs de moins de 18 ans.

Au fur et à mesure que se déploie la question du règlement européen de ce dossier, on note une diminution des articles virulents sur les pratiques " négrières ». Il reste à interroger ce double mouvement. On peut considérer que ces deux séries (disparition de la critique concernant les pratiques clandestines / émergence progressive du thème du «système international de transferts de footballeurs ") sont partiellement indépendantes. Les médias choisiraient de traiter du deuxième thème afin de renouveler leurs contenus d'information et de s'assurer ainsi une meilleure audience. On peut également penser qu'à la suite du rapport Donzel, le dossier africain se reconfigure pour être abordé d'une façon plus globale :après la polémique provoquée par l'enquête ministérielle, la question qui s'impose serait alors moins celle du cas de migrants d'un continent vers un pays européen que celle portant sur des transferts de joueurs mineurs circulant au sein de différents États. On peut aussi interpréter cette évolution comme l'aboutissement d'un travail entrepris suite au problème dénoncé. Les décisions européennes apparaîtraient comme la réponse appropriée pour réguler des pratiques mises en évidence par le rapport et les acteurs auraient réussi à identifier le niveau pertinent d'intervention publique. Enfin, on peut aussi supposer qu'il s'agit essentiellement d'une volonté politique visant à la fois à réguler le football et à laisser une place décisive aux pouvoirs publics dans leur mode d'intervention et de définition de la notion de "spécificité du sport».

Nous ne pouvons pas trancher sur la validité et la justesse de ces interprétations. Pour élucider la question, il serait utile de s'intéresser aux raisons pour lesquelles la presse a peu à peu abandonné un dossier pour en privilégier un autre. Or, pour réaliser ce travail, il faudrait interroger les journalistes. Il s'agit là d'une limite de notre étude, et plus généralement, de nombreuses recherches sur les affaires sportives quand elles laissent les articles des journalistes parler pour eux et ne les interviewent pas.

\section{La presse aux prises avec le sport}

Lors du Tour de France 98, les journalistes ont assez vite délaissé les événements sportifs pour s'intéresser à l'affaire dite Festina. Ils ont emboîté le pas à l'institution judiciaire et profité du succès des policiers pour réaliser des interviews visant à révéler les pratiques du peloton, pour mener leurs enquêtes, pour recouper des témoignages et, ainsi, pour organiser un travail visant à faire éclater la vérité sur l'ampleur du dopage, qu'ils se sont pendant longtemps employés à dénoncer.

Le rôle de la presse dans le dossier de 
l'immigration des footballeurs africains est bien différent.Les journalistes restent proches des informations contenues dans un rapport confidentiel publié dans Le Parisien. Les enquêtes ne revien-nent qu'à décrire la situation des "vic-times" identifiées dans le rapport et à donner la parole à ceux que ce même texte désigne comme les responsables. Les médias s'emploient surtout à rendre public les discussions autour des méthodes d'investigations,sans travailler directement à établir des faits nouveaux. La véracité et l'ampleur des pro-blèmes au cœur de ces dossiers méri-tent une attention particulière, à laquelle les journalistes consacrent une grande partie de leur activité.Eux aussi s'affairent à resituer les enjeux des dos-siers. Qu'il s'agisse de replacer l'affaire Festina dans une perspective histo-rique marquée par des secrets, des soupçons et des défaites judiciaires, ou, dans le cas des footballeurs africains, de replacer l'action de Marie-George Buffet dans la politique européenne de lutte contre l'immigration clandestine, l'activité journalistique est marqué par

\section{Notes}

1 On peut lire en titre, par exemple: «MarieGeorge Buffet veut abolir l'esclavagisme dans le foot " (La Croix, 14 février 2000).

2 Quatorze pour la presse généraliste nationale et régionale (Libération, Ouest-France, etc...), trois articles émanant de bulletins d'information des pouvoirs publics (Relais JS), trois organes sportifs (L'Equipe, La Lettre du sport, La Lettre de l'économie du Sport), trois jour- un travail interprétatif qui, sous des contraintes d'originalité (Lemieux 2000), pèsent lourdement dans le traitement de l'information.

Cette étude sur les façons dont la presse travaille le sport mériterait d'être approfondie et poursuivie. Et ce, pour des raisons qui dépassent la stricte analyse du fonctionnement des médias. Dans les objets traités par les sciences sociales, il semble décisif, en effet, de saisir comment cet acteur participe à l'affaire lorsqu'il la relie à une histoire, à des faits semblables dans différents pays, à d'autres dossiers. La façon dont les journalistes mènent l'enquête (Dewey, 1993) et les processus de construction des jugements sont au cœur du programme pragmatique. "Comment considérons-nous les choses comme réelles?» se demandait William James (1998). Il semble qu'une analyse plus systématique des médiations des journalistes, entendues comme le déploiement des éléments de l'enquête, constituerait un programme de recherches heuristique dans l'étude des disputes.

naux spécialisés dans le football (France Football, Foot Magazine, Giga Foot)

3 Voir le site Internet présentant l'outil: www.prosperologie.org, ainsi que l'ouvrage en explicitant les principes (Chateauraynaud, 2003).

4 Le travail réalisé ici n'est pas lexicométrique au sens linguistique du terme, puisqu'il repose sur des opérations de rassemblement sémantique des entités, assumées et contrôlées par le sociologue.Cf.l'annexe méthodologique.Par 
" entité ", nous entendons autant les noms propres et communs du corpus que les groupes nominaux définis par le chercheur. Voir le chapitre 8 de Chateauraynaud (2003).

5 Fédération française de cyclisme, Union cycliste internationale,Amaury Sports Organisation qui est propriétaire du Tour de France.

6 Sur la dimension dramatique des événements dans lesquels sont engagés les sportifs, on peut se reporter à Duret (1993).

7 On lui reproche une "complicité de facilitation, d'incitation à usage et d'administration à autrui de substances ou de procédés de nature à modifier artificiellement les capacités ou à masquer l'emploi de substances ou procédés ayant cette propriété au cours des compétitions et manifestations sportives organisées ou agréées par des fédérations sportives ou en vue d'y participer: délits prévus et réprimés par les articles 1 et 14 de la loi du 28 juin 1989, articles 121-6 et 121-7 du code pénal».

8 On lit par exemple dans Libération: «Pour ce même JT, le service des sports avait proposé une interview de RichardVirenque. Mais sous certaines conditions. Béatrice Schönberg, la présentatrice, prévient que toutes les questions, même celles qui fâchent, seront posées. Du coup, pas deVirenque.» (27 juillet 1998).

9 Les différends sont assez classiques: des confidences données en fin d'interview et rendues publiques à tort (les menaces de Virenque envers Baal narrées à un journaliste réunionnais, comme le raconte Guillon dans

\section{Bibliographie}

Boltanski, L. (1990). L'Amour et la justice comme compétences. Trois essais de sociologie de l'action (Métailié ed.). Paris.

Boltanski, L., Darré, Y., \& Schiltz, M.-A. (1984). "La dénonciation ». Actes de la recherche en sciences sociales, 51, pp.3-40.

Boltanski, L., \& Thévenot, L. (1991). De la son ouvrage), un invité non informé de la présence sur le plateau d'un contradicteur (Killy /Delion sur France 2, raconté par Libération du 27 juillet 1998)...

10 Les catégories d'entités qui émergent de la version publique du rapport Donzel font référence à l'immigration et aux centres de formation "parallèles ». Le régime de polémique est quasiment absent du rapport, ce qui tend également à le distinguer du sous-corpus presse. Jacques Donzel prend la précaution d'énoncer systématiquement ses critiques évaluatives à partir de constats.

11 Les autres victimes en France, qui prennent la parole dans la presse sontVictor, François X, Saliou Lassissi et Issiaga Condé.

12Tshimanga Bakadiababu,E. (2001). Le commerce et la traite des footballeurs africains et sudaméricains en Europe (L'Harmattan ed.). pp.273-278.

13 Lien en consultation interne au Ministère http://www.jeunesse-sports.gouv.fr/ francais/disc140200.htm

14 Riche, M., (1998), Le rêve africain: La star d'ébène. Film documentaire, Coproduction Canal+, RTBF, Gédéon Programmes, Périscope Productions. Distribution ADAV/ RTBF, VHS SECAM. 54 minutes. Le réalisateur filme "in-vivo ", à la manière de la célèbre émission «strip-tease » des tranches de vie de la relation entre un jeune footballeur nigérian et son agent depuis le départ de son pays à la signature de son premier contrat dans un club amateur d'une banlieue belge.

justification. Les économies de la grandeur (Gallimard ed.), Paris.

Buisine, S. (2007). «Le métier de cycliste professionnel: les contraintes de la rémunération "In Le sport: entre intérêts publics et appropriations privées (L'Harmattan / Société de sociologie du sport de langue française, ed.), à paraître. 
Chateauraynaud, F. (1991). La Faute professionnelle: Une sociologie des conflits de responsabilité. (Métailié ed.). Paris.

Chateauraynaud, F., \& Torny, D. (1999). Les sombres précurseurs : une sociologie pragmatique de l'alerte et du risque. (EHESS ed.). Paris.

Dewey,J. (ed.). (1993). Logique. La Théorie de l'enquête (PUF ed.). Paris.

Duret, P., \& Trabal, P. (2001). Le Sport et ses affaires. Une sociologie de la justice de l'épreuve sportive (Métailié ed.). Paris.

James, W. (1998). La Signification de la vérité (Antipodes ed.). Lausanne.

Latour, B. (1989). La Science en action (La Découverte ed.). Paris.

Lemieux, C. (2000). Mauvaise presse. Une Sociologie compréhensive du travail journalistique et de ses critiques. (Métailié ed.). Paris.
Torny, D. (2001). Un seul cas suffit. Une étude sociologique de l'engagement du futur en biomédecine, Thèse de doctorat en sociologie. (EHESS ed.). Paris.

Torny, D, \& Trabal, P. (2007). «Toutes choses égales par ailleurs. Comparer deux congrès de l'Association Française de Sociologie ", BMS, (l'Harmattan ed.), Paris, avril 2007, 94, pp.57-75.

Torny, D., \& Trabal P., (2006). «Le résumé de communication comme objet sociologique - une analyse thématique, ontologique et littéraire à l'aide du logiciel Prospéro » in Demazière, D., Brossaud, C., Trabal, P., \&Van Meter, K.,(dir.). Analyses textuelles en sociologie. PUR, pp.23-80.

Trabal, P., (2006). « Les approches pragmatiques et la sociologie du sport ", In Sociologie du Sport - débats et critiques (L'Harmattan Ed.), pp.57-64.

\section{Annexe méthodologique Un exemple de travail d'indexation et de codification avec le logiciel Prospéro ${ }^{\circledR}$}

La démarche explicitée ici permet de saisir la façon dont nous avons, plus généralement travaillé notre corpus. Nous avons créé une série de "concepts " dans le logiciel Prospéro, c'est-à-dire un ensemble de syntagmes construit par le chercheur selon sa problématique de recherche et de la nature du corpus. Contrairement à d'autres logiciels d'analyse textuelle, Prospéro n'impose pas une seule méthode de travail (Torny \& Trabal,2006), mais un ensemble de chemins (Chateauraynaud, 2003). La démarche proposée ici n'est qu'un exemple de raisonnement rendu possible par la machine.

Un exemple: la catégorie d'entités "Figures de dénonciation des transferts", regroupe des termes comme "pillage",
" drôle de marché ", " affaires d'exploitation des jeunes mineurs ".... De même, nous avons rassemblé sous la dénomination " centre de formation parallèle " contient "Structures parallèles », « établissements non agréés ", "structures périphériques "...

Nous avons ensuite généré un corpus de textes afin de déterminer les énoncés contenant simultanément ces deux concepts afin de recenser de façon systématique les critiques liées à ce type d'institutions. Nous avons alors relevé les différences et les similitudes entre les sous-corpus. Le travail a consisté à regarder comment des entités, des thèmes et d'autres catégories émergent ou non dans tel ou tel type de support tout en tenant compte de la variable temporelle. 